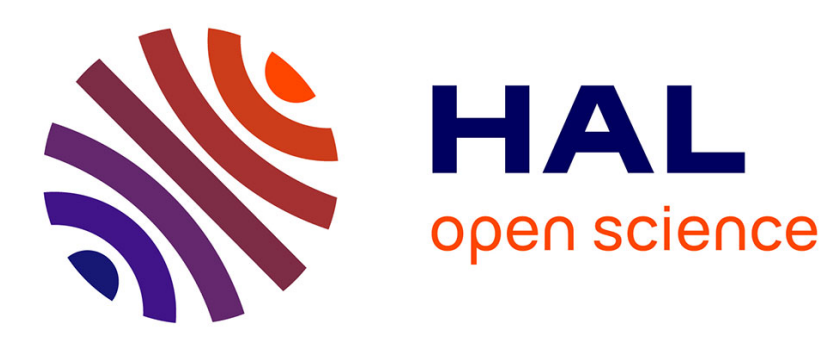

\title{
An Iterative Method for Solving Non-Linear Hydromagnetic Equations
}

\author{
Cédric Boulbe, Tahar Zamène Boulmezaoud, Tahar Amari
}

\section{To cite this version:}

Cédric Boulbe, Tahar Zamène Boulmezaoud, Tahar Amari. An Iterative Method for Solving NonLinear Hydromagnetic Equations. ENUMATH 2005, Jul 2005, France. pp.917-925, 10.1007/978-3540-34288-5 . hal-00436505

\section{HAL Id: hal-00436505 https://hal.science/hal-00436505}

Submitted on 26 Nov 2009

HAL is a multi-disciplinary open access archive for the deposit and dissemination of scientific research documents, whether they are published or not. The documents may come from teaching and research institutions in France or abroad, or from public or private research centers.
L'archive ouverte pluridisciplinaire HAL, est destinée au dépôt et à la diffusion de documents scientifiques de niveau recherche, publiés ou non, émanant des établissements d'enseignement et de recherche français ou étrangers, des laboratoires publics ou privés. 


\section{An iterative method for solving non-linear hydromagnetic equations}

C. BOULBE ${ }^{1}$, T.Z. BOULMEZAOUD ${ }^{2}$, and T. AMARI ${ }^{3}$

1 Laboratoire de mathématiques appliquées, Université de Pau et des Pays de l'Adour, IPRA - Av de l'Université, 64000 PAU, FRANCE.

c.boulbe@etud.univ-pau.fr

2 Laboratoire de mathématiques appliquées, Université de Versailles Saint Quentin, 45 av des Etats Unis, 75035 Versailles, FRANCE. boulmeza@math.uvsq.fr

3 Centre de Physique Théorique, Ecole Polytechnique, F91128 Palaiseau Cedex,FRANCE. amari@cpht.polytechnique.fr

Summary. We propose an iterative finite element method for solving non-linear hydromagnetic and steady Euler's equations. Some three-dimensional computational tests are given to confirm the convergence and the high efficiency of the method.

\section{Introduction. Statement of the problem}

The understanding of plasma equilibria is one of the most important problems in magnetohydrodynamics and arises in several fields including solar physics and thermonuclear fusion. Such an equilibria is often governed by the well known steady hydromagnetic equations

$$
\begin{array}{r}
\operatorname{curl} \mathbf{B} \times \mathbf{B}+\nabla p=0, \\
\operatorname{div} \mathbf{B}=0,
\end{array}
$$

which describe the balance of the Lorentz force by pressure. Here $\mathbf{B}$ and $p$ are respectively the magnetic field and the pressure.

Notice that system (1) $+(2)$ is quite similar to steady inviscid fluid equations

$$
\begin{array}{r}
\mathbf{v} \cdot \nabla \mathbf{v}+\nabla p=0 \\
\operatorname{div} \mathbf{v}=0 .
\end{array}
$$

This analogy is due to the vectorial identity 


$$
\mathbf{v} . \nabla \mathbf{v}-\nabla \frac{|\mathbf{v}|^{2}}{2}=\operatorname{curl} \mathbf{v} \times \mathbf{v}
$$

System of equations (1) $+(2)$ must be completed with some boundary conditions on $\mathbf{B}$ and $p$. Physical considerations suggest to prescribe the boundary normal field component:

$$
\text { B.n }=g \text { on } \partial \Omega
$$

where $g$ satisfies the compatibility condition $\int_{\Omega} g=0$ due to the equation $\operatorname{div} \mathbf{B}=0$. Defining the inflow boundary as $\Gamma^{-}=\{\mathbf{x} \in \Omega, \mathbf{B}(\mathbf{x}) \cdot \mathbf{n}(\mathbf{x})<0\}$, one can also prescribe the normal component curl B.n of the current density and the pressure $p$ on $\Gamma^{-}$

$$
\operatorname{curl} \text { B.n }=h \text { on } \Gamma^{-} \text {, }
$$

$$
p=p_{0} \text { on } \Gamma^{-} .
$$

One can notice that if the pressure is neglected, equations (1) $+(2$ become

$$
\begin{array}{r}
\operatorname{curl} \mathbf{B} \times \mathbf{B}=\mathbf{0}, \\
\operatorname{div} \mathbf{B}=0 .
\end{array}
$$

Equation (8) means that the magnetic field and its curl, which represents the current density, are everywhere aligned. The magnetic field is said Beltrami or force-free $(\mathrm{FF})$. A usual way to tackle the problem $(8)+(9)$ consists to rewrite equation (8) into the form

$$
\operatorname{curl} \mathbf{B}=\lambda(\mathbf{x}) \mathbf{B},
$$

where $\lambda(\mathbf{x})$ is a scalar function which can be a constant function or can depend on $\mathbf{x}$. In the former, the $\mathbf{B}$ field is said linear FF. In the latter, it is said non linear.

Some partial results concerning existence of 3D solutions of equations (11) $+(2)$ in bounded domains are given in [1] and [11]. Linear force-free-fields were studied in [4]. For the existence of non-linear ones the reader can refer to [5], [3].

The numerical solving of equations (12) $+(2)$ and equations (8) $+(9)$ is of importance in magnetohydrodynamics studies and in solar physics. As it is known, the reconstruction of the coronal magnetic field has is of a great utility in observational and theoretical studies of the magnetic structures in the solar atmosphere. In this paper, we propose an iterative process for solving these equations (section 2). A finite element method is proposed for solving each one of the arising problems. 


\section{An iterative method for the magnetostatic system}

Our objective here is to expose an iterative method for solving the non-linear equations (11) +(2) in a bounded and simply-connected domain. The starting idea of the method consists to split the current density $\boldsymbol{\omega}=\operatorname{curl} \mathbf{B}$ into the sum

$$
\boldsymbol{\omega}=\boldsymbol{\omega}_{\|}+\boldsymbol{\omega}_{\perp},
$$

where the vector field $\boldsymbol{\omega}_{\|}=\mu(\mathbf{x}) \mathbf{B}$ is collinear to $\mathbf{B}$, while $\boldsymbol{\omega}_{\perp}$ is perpendicular to $\mathbf{B}$. The problem is decomposed formally into a curl-div system on $\mathbf{B}(\mathbf{x})$ and two first order hyperbolic equations on $\mu(\mathbf{x})$ and $p(\mathbf{x})$.

More precisely, writing $\boldsymbol{\omega}_{\|}(\mathbf{x})=\mu(\mathbf{x}) \mathbf{B}(\mathbf{x})$ where $\mu$ is a scalar function and taking the divergence of (11), gives

$$
\text { B. } \nabla \mu=-\operatorname{div} \omega_{\perp} \text {. }
$$

Notice that the pressure satisfies a similar equation since

$$
\text { B. } \nabla p=0 \text {. }
$$

Equation (11) becomes

$$
\boldsymbol{\omega}_{\perp} \times \mathbf{B}=-\nabla p
$$

which means that $\boldsymbol{\omega}_{\perp}(\mathbf{x})=\frac{1}{|\mathbf{B}(\mathbf{x})|^{2}} \nabla p(\mathbf{x}) \times \mathbf{B}(\mathbf{x})$ if $|\mathbf{B}(\mathbf{x})| \neq \mathbf{0}$.

In consideration of these remarks, we are going now to propose an iterative process to solve non-linear systems (11) + (2). In this process the transport equation (12) is perturbed by adding an artificial reaction term. Namely, we construct a sequence $\left(\mathbf{B}^{(n)}, p^{(n)}\right)_{n \geq 0}$ as follows:

- The starting guess $\mathbf{B}_{0} \in H^{1}(\Omega)$ is chosen as the irrotational field associated to $g$ defined by

$$
\operatorname{curl} \mathbf{B}_{0}=\mathbf{0} \text { in } \Omega, \operatorname{div} \mathbf{B}_{0}=0 \text { in } \Omega \text { and } \mathbf{B}_{0} \cdot \mathbf{n}=g \text { on } \partial \Omega .
$$

This is a usual problem which can be reduced to a scalar Neumann problem since the domain is simply-connected.

- For all $n \geq 0, p^{(n)}$ is solution of the system

$$
\left\{\begin{aligned}
\mathbf{B}^{(n)} \cdot \nabla p^{(n)}+\eta p^{(n)} & =\eta p^{(n-1)} \text { in } \Omega \\
p^{(n)} & =p_{0} \text { on } \partial \Omega
\end{aligned}\right.
$$

where $\eta$ is a small parameter and $p^{(-1)}=0$.

- For all $n \geq 0, \boldsymbol{\omega}_{\perp}{ }^{(n)}=\frac{1}{\left|\mathbf{B}^{(n)}\right|^{2}} \nabla p^{(n)} \times \mathbf{B}^{(n)}$ and $\boldsymbol{\omega}_{\|}{ }^{(n)}=\mu^{(n)} \mathbf{B}^{(n)}$, where $\mu^{(n)}$ satisfies

$$
\left\{\begin{aligned}
\mathbf{B}^{(n)} \cdot \nabla \mu^{(n)}+\epsilon \mu^{(n)} & =-\operatorname{div} \boldsymbol{\omega}_{\perp}{ }^{(n)}+\varepsilon \mu^{(n-1)} \text { in } \Omega, \\
\mu^{(n)}\left(\mathbf{B}^{(n)} \cdot \mathbf{n}\right) & =h-\boldsymbol{\omega}_{\perp}{ }^{(n)} \cdot \mathbf{n} \text { on } \Gamma^{-} .
\end{aligned}\right.
$$

Here $\mu^{(-1)}=0$ and $\epsilon$ is a small parameter. 
- For all $n \geq 0, \mathbf{B}^{(n+1)}=\mathbf{B}_{0}+\mathbf{b}^{(n+1)}$, with $\mathbf{b}^{(n+1)}$ solution of

$$
\left\{\begin{array}{l}
\operatorname{curl} \mathbf{b}^{(n+1)}=\boldsymbol{\omega}^{(n)}+\nabla q^{(n)} \text { in } \Omega, \\
\operatorname{div} \mathbf{b}^{(n+1)}=0 \text { in } \Omega \\
\mathbf{b}^{(n+1)} \cdot \mathbf{n}=0 \text { on } \partial \Omega
\end{array}\right.
$$

where $\boldsymbol{\omega}^{(n)}=\boldsymbol{\omega}_{\|}{ }^{(n)}+\boldsymbol{\omega}_{\perp}{ }^{(n)}$ while $q^{(n)}$ is solution of the Laplace problem

$$
-\Delta q^{(n)}=\operatorname{div} \boldsymbol{\omega}^{(n)} \text { in } \Omega, \text { and } q^{(n)}=0 \text { on } \partial \Omega .
$$

Notice that the appearance of the correction term $\nabla q^{(n)}$ is due to the fact that $\operatorname{div}\left(\boldsymbol{\omega}^{(n)}\right)$ is not zero in general.

The convergence of this iterative process is not an easy matter. We conjecture that it converges if $h$ is sufficiently small and $\left|\mathbf{B}_{0}(\mathbf{x})\right| \geq c>0$ in $\Omega$ for some constant $c>0$. Nevertheless, in the case of linear force-free fields (in that case the algorithm is simplified since at each iteration $p^{(n)}=0, \boldsymbol{\omega}_{\perp}{ }^{(n)}=\mathbf{0}$ and $\mu^{(n)}$ is a fixed real) Boulmezaoud and Amari [6] proved that this process is super-convergent. The proof of convergence in the general case is not given and remains an open question.

Notice that the same algorithm can be used for computing linear or non-linear force-free fields which are solutions of (9) +10 , provided that the computation of the pressure $p^{(n)}$ and the vector field $\boldsymbol{\omega}_{\perp}{ }^{(n)}$ are dropped.

\section{Finite element discretization}

Here we give a short description of the finite elements methods we use for solving problems arising in the iterative process exposed above. Observe first that at each iteration of the algorithm one should solve two problems:

(a) A reaction-convection problem of the form: find u solution of

$$
\left\{\begin{aligned}
\operatorname{div}(u \mathbf{B})+\sigma u & =f \text { in } \Omega, \\
u & =h \text { on } \Gamma^{-} .
\end{aligned}\right.
$$

(b) A vector potential problem: find the pair $(\mathbf{b}, q)$ satisfying

$$
\left\{\begin{aligned}
\operatorname{curl} \mathbf{b}-\nabla q=\mathbf{j} & \text { in } \Omega, \\
\operatorname{div} \mathbf{b}=0 & \text { in } \Omega, \\
\mathbf{b . n}=0 & \text { on } \partial \Omega, \\
q=0 & \text { on } \partial \Omega .
\end{aligned}\right.
$$

We begin with the approximation of (19).

It is well known that the direct application of a Galerkin finite elements method to the singularly perturbed problem (19) may lead to the appearance of spurious oscillations and instabilities. In the two last decades, several methods were proposed to remove this drawback (especially in the two dimensional 
case). Among these methods, one can recall the streamline diffusion method (see Brookes and Hughes [8], see also, e. g., Johnson et al. [10]), the discontinuous Galerkin method (see Lesaint 12]) and bubble functions methods (see, e. g., Brezzi et al. [7]). Here we shall use the method of streamline diffusion.

Thus, let us consider a family of regular triangulations $\left(\mathscr{T}_{h}\right)$ of $\Omega$. The discrete problem we consider is

$$
\left(\mathscr{P}_{h}\right)\left\{\begin{array}{l}
\text { Find } u_{h} \in W_{h} \text { such that } \\
a_{h}\left(u_{h}, w_{h}\right)=\ell_{h}\left(w_{h}\right), \forall w_{h} \in W_{h},
\end{array}\right.
$$

where

$$
\begin{aligned}
a_{h}\left(u_{h}, w_{h}\right) & =\int_{\Omega}\left(\mathbf{B} \cdot \nabla u_{h}+\sigma u_{h}\right) \cdot\left(w_{h}+\delta_{h} \mathbf{B} \cdot \nabla w_{h}\right) d x-\int_{\Gamma^{-}} u_{h} w_{h}(\mathbf{B} \cdot \mathbf{n}) d x, \\
\ell_{h}\left(w_{h}\right) & =\int_{\Omega} f(\mathbf{x})\left(\mathbf{B} \cdot \nabla w_{h}+\delta_{h} w_{h}\right)-\int_{\Gamma^{-}} \alpha_{0} w_{h}(\mathbf{B} \cdot \mathbf{n}) d x .
\end{aligned}
$$

Here $W_{h}$ stands for the finite elements space

$$
W_{h}=\left\{v_{h} \in H^{1}(\Omega) ; v_{\mid K} \in \mathbb{P}_{k}(K), \forall K \in \mathscr{T}_{h}\right\},
$$

where for each $K \in \mathscr{T}_{h}, \mathbb{P}_{k}(K)$ denotes the space of polynomials of degree less or equal $k$.

One can prove that the problem $\left(\mathscr{P}_{h}\right)$ has a unique solution $u_{h} \in W_{h}$ when $\delta_{h} \sigma<1$. Moreover, if $\delta_{h}=c h$ for some constant $c$ and if $\mathbf{B} \in L^{\infty}(\Omega)^{3} \cap$ $H(\operatorname{div} ; \Omega)$ and $u \in H^{\ell+1}(\Omega)$ for some $\ell \geq 1$, then

$$
\left(1-\delta_{h} \sigma\right)\left\|\left|u-u_{h}\left\|\mid \leq C h^{\ell+1 / 2}\right\| u \|_{H^{\ell+1}(\Omega)},\right.\right.
$$

where $\left\|\left|w\|\|_{\Omega}^{2}=\delta_{h}\|\mathbf{B} . \nabla w\|_{L^{2}(\Omega)}^{2}+\sigma\|w\|_{L^{2}(\Omega)}^{2}+\left\|\left.\mathbf{B} \cdot \mathbf{n}\right|^{1 / 2} w\right\|_{L^{2}(\partial \Omega)}^{2}\right.\right.$.

Now, we deal with the approximation of the curl-div system (20), which can be dispatched into two problems: a variational problem $(\mathscr{Q})$ in terms of $\mathbf{b}$ and the fictitious unknown $\theta=0$, and Laplace equation (18) in terms of $q$. We only deal with the approximation of $\mathbf{b}$, since we shall see that the computation of the $q$ is useless. Denote by $H(\mathbf{c u r l} ; \Omega)$ the space

$$
H(\operatorname{curl} ; \Omega)=\left\{\mathbf{v} \in L^{2}(\Omega)^{3} ; \operatorname{curl} \mathbf{v} \in L^{2}(\Omega)^{3}\right\}
$$

equipped with its usual norm. The statement of problem (20) suggests the use of an $H(\operatorname{curl} ; \Omega)$ approximation. Define $M$ the space

$$
M=\left\{v \in H^{1}(\Omega), \quad \int_{\Omega} v d x=0\right\} .
$$

Let $X_{h} \subset H(\operatorname{curl} ; \Omega), M_{h} \subset M$ two finite-dimensional subspaces and set

$$
V_{h}=\left\{\mathbf{v}_{\mathbf{h}} \in X_{h} ;\left(\mathbf{v}_{\mathbf{h}}, \nabla \mu_{h}\right)=0, \forall \mu_{h} \in M_{h}\right\} .
$$

We make the following assumptions 
$\left(\mathscr{H}_{1}\right)$ the inclusion $\left\{\nabla \mu_{h}, \mu_{h} \in M_{h}\right\} \subset X_{h}$ holds,

$\left(\mathscr{H}_{2}\right)$ there exists a constant $C$ such that

$$
\left\|\mathbf{v}_{\mathbf{h}}\right\|_{0, \Omega} \leq C\left\|\mathbf{c u r l} \mathbf{v}_{\mathbf{h}}\right\|_{0, \Omega}, \forall \mathbf{v}_{\mathbf{h}} \in V_{h} .
$$

The discrete version of problem (20) writes

$$
\left(\mathscr{Q}_{h}\right)\left\{\begin{array}{l}
\text { Find }\left(\mathbf{b}_{\mathbf{h}}, \theta_{h}\right) \in X_{h} \times M_{h} \text { such as } \\
\forall \mathbf{v}_{\mathbf{h}} \in X_{h}, \int_{\Omega} \mathbf{c u r l} \mathbf{b}_{\mathbf{h}} \cdot \mathbf{c u r l} \mathbf{v}_{\mathbf{h}} d x+\int_{\Omega} \mathbf{v}_{\mathbf{h}} \cdot \nabla \theta_{h} d x=\int_{\Omega} \mathbf{j} \cdot \operatorname{curl} \mathbf{v}_{\mathbf{h}} d x \\
\forall \mu_{h} \in M_{h}, \int_{\Omega} \mathbf{b}_{\mathbf{h}} \cdot \nabla \mu_{h}=0 .
\end{array}\right.
$$

According to Amrouche and al. [2], the problem $\left(\mathscr{Q}_{h}\right)$ has one and only one solution $\left(\mathbf{b}_{h}, \theta_{h}\right)$ with $\theta_{h}=0$, and

$$
\left\|\mathbf{b}-\mathbf{b}_{\mathbf{h}}\right\|_{H(\operatorname{curl} ; \Omega)} \leq C \inf _{\mathbf{v}_{\mathbf{h}} \in X_{h}}\left\|\mathbf{b}-\mathbf{v}_{\mathbf{h}}\right\|_{H(\operatorname{curl} ; \Omega)} .
$$

A simple manner for constructing the spaces $X_{h}$ and $M_{h}$ is to use the $H(\mathbf{c u r l})$ conforming elements of Nédelec 13. (see Amrouche and al.). In that case, the following estimate holds

$$
\left\|\mathbf{b}-\mathbf{b}_{\mathbf{h}}\right\|_{H(\mathbf{c u r l} ; \Omega)} \leq C h^{\ell}\left\{|\mathbf{b}|_{\ell, \Omega}+|\mathbf{b}|_{\ell+1, \Omega}\right\}
$$

which is valid if $\mathbf{b} \in H^{\ell+1}(\Omega)$.

An important feature of the discrete system $\left(\mathscr{Q}_{h}\right)$ is that only the discrete vector field $\mathbf{b}_{\mathbf{h}}$ is really unknown. Actually, we know that $\theta_{h}=0$. This property can be exploited from a practical viewpoint to reduce the discrete system to a smaller one by eliminating $\theta_{h}$. In term of matrices, the system writes

$$
\left(\begin{array}{cc}
A^{\text {curl }} & B^{T} \\
B & 0
\end{array}\right)\left(\begin{array}{l}
X \\
Y
\end{array}\right)=\left(\begin{array}{c}
C^{\text {curl }} \\
0
\end{array}\right)
$$

where $A^{\text {curl }}$ is a symmetric and positive square matrix ( $A^{\text {curl }}$ is not definite neither invertible). We can state the following

Lemma 1. Let $\Lambda$ be a square positive, definite and symmetric matrix having the same size as $A$. Then, the pair $(X, Y)$ is solution of (24) if and only if $Y=0$ and $X$ is solution of

$$
\left(A^{\text {curl }}+B^{T} \Lambda B\right) X=C^{\text {curl }} .
$$

Remark 1. In Lemma1, the matrix $A^{\text {curl }}$ and the RHS $C^{\text {curl }}$ are not arbitrary. Indeed, if $G$ denotes the matrix of the operator $\nabla: M_{h} \rightarrow X_{h}$, then necessarily $G^{T} A^{\text {curl }}=0$ and $G^{T} C^{\text {curl }}=0$. These identities are the discrete counterpart of the continuous relations $\operatorname{div}(\mathbf{c u r l})=$.0 and $\operatorname{div} \mathbf{j}=0$.

A serious advantage of the new system (25) comparing with (24) is that number of unknowns is reduced. 


\section{Computational tests}

In this last section, we expose some computational results we obtain with a 3D code. This code use the iterative method and the finite elements discretization exposed above to solve problem (11) $+(2)$ and problem (10) $+(9)$. We compare the exact solution and the numerical solution and we show the behavior of the errors in terms of $h$. Two exact solutions are used for the tests.

\section{- Test 1 (a non-linear force-free-field).}

Let $(r, \theta, z)$ the cylindrical coordinates with respect to a point $\left(x_{0}, y_{0}, 0\right)$ $\left(x_{0}=-3\right.$ and $\left.y_{0}=-3\right)$. The pair $(\mathbf{B}, p)$ is given $\mathbf{B}=\frac{1}{\sqrt{r}}\left(\mathbf{e}_{\theta}+\mathbf{e}_{z}\right), p(\mathbf{x})=$ 0 . This is a non-linear force-free field with $\lambda=\frac{1}{2 r}$. Table 1 shows the behavior of the residue $\left\|\mathbf{B}^{(n+1)}-\mathbf{B}^{(n)}\right\|_{0, \Omega}$ and the product $\operatorname{curl} \mathbf{B}^{(n)} \times \mathbf{B}^{(n)}$ versus the iteration number. This example illustrates the superconvergence of the algorithm.

Table 1. Evolution of $\frac{\left\|\mathbf{B}^{(n+1)}-\mathbf{B}^{(n)}\right\|_{0, \Omega}}{\left\|\mathbf{B}^{(n)}\right\|_{0, \Omega}}$ and $\left\|\operatorname{curl} \mathbf{B}^{(n)} \times \mathbf{B}^{(n)}\right\|_{\infty}$.

\begin{tabular}{lll}
\hline$n \frac{\left\|\mathbf{B}^{(n+1)}-\mathbf{B}^{(n)}\right\|_{0, \Omega}}{\left\|\mathbf{B}^{(n)}\right\|_{0, \Omega}}$ & $\left\|\mathbf{c u r l} \mathbf{B}^{(n)} \times \mathbf{B}^{(n)}\right\|_{\infty}$ \\
\hline 0 & 0.09912 & $6.740 \mathrm{e}-15$ \\
1 & 0.00566 & 0.06781 \\
2 & 0.00036 & 0.01939 \\
3 & $2.644 \mathrm{e}-05$ & 0.01910 \\
\hline
\end{tabular}

- Test 2: (Bennet pinch). The pair $(\mathbf{B}, p)$ is given by

$$
\mathbf{B}=\nabla A \times \mathbf{e}_{y} \text { and } p=\frac{\lambda}{2} \mathrm{e}^{2 A} \text { with } A=-\ln \left(\frac{1+\lambda k^{2}\left(x^{2}+z^{2}\right)}{2 k}\right) .
$$

In table 2, the relative $L^{2}$ errors on $\mathbf{B}$ and $p$ after convergence of the algorithm are shown. These error decreases as $h^{1.8}$, which confirms the high accuracy of the method.

Table 2. Relative errors on $\mathbf{B}_{h}$ and $p_{h}$ in norm $L^{2}$ (test 2).

\begin{tabular}{lcc}
\hline$h$ & $\frac{\left\|\mathbf{B}-\mathbf{B}_{h}\right\|_{0, \Omega}}{\|\mathbf{B}\|_{0, \Omega}}$ & $\frac{\left\|p-p_{h}\right\|_{0, \Omega}}{\|p\|_{0, \Omega}}$ \\
\hline 0.69282 & 0.03837 & 0.08648 \\
0.23094 & 0.00492 & 0.01102 \\
0.13856 & 0.00191 & 0.00396 \\
0.09897 & 0.00108 & 0.00201 \\
\hline
\end{tabular}



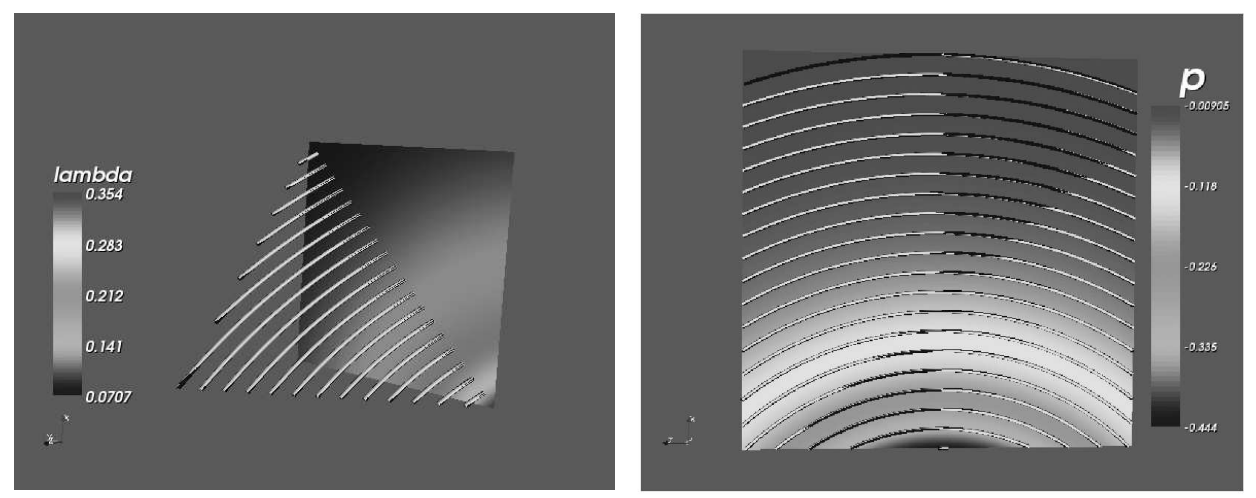

Fig. 1. Superposition of the the exact and the numerical solutions in the case of test 1 on the left and in a $(x-z)$ plane $2 \mathrm{D}$ cut for the test 2 on the right.

\section{References}

1. Alber, H.D.: Existence of three-dimensional, steady, inviscid, incompressible flows with non-vanishing vorticity. Math. Ann. 292, 493-528 (1992)

2. Amrouche, C., Bernardi, C ., Dauge, M., Girault, V.: Vector potentials in threedimensional non-smooth domains, Math. Methods Appl. Sci. 9, 823-864 (1998).

3. Boulmezaoud, T.Z.: On the existence of non-linear Beltrami fields. Comptes Rendus de l'Académie des Sciences, 328, 437-442 (1999).

4. Boulmezaoud, T.Z., Maday, Y., Amari, T.: On the linear Beltrami fields in bounded and unbounded three-dimensional domains: Mathematical Modelling and Numerical Analysis, 33, 359-394 (1999)

5. Boulmezaoud, T.Z. , Amari, T.: On the existence of non-linear force-free fields in three-dimensional multiply-connected domains. à paraître dans Zeitschrift für Angewandte Mathematik und Physik (ZAMP).

6. Boulmezaoud, T. Z., Amari, T.: Approximation of linear force-free fields in bounded 3-D domains. Math. Comput. Modelling 31, 109-129 (2000)

7. Brezzi, F., Hauke, G., Marini, L.D., Sangalli, G.: Link-Cutting Bubbles for the Stabilization of Convection-Diffusion-Reaction Problems, Math. Models Methods Appl. Sci., 13, 445-461 (2003)

8. Brooks, A., Hughes,T. R. J.: Streamline upwind/Petrov-Galerkin formulations for convection dominated flows with particular emphasis on the incompressible Navier-Stokes equations . FENOMECH '81, Part I (Stuttgart, 1981). Comput. Methods Appl. Mech. Engrg. 32, 199-259 (1982).

9. Girault, V., Raviart, P. A.: Finite element methods for Navier-Stokes equations. Springer-Verlag (1986).

10. Johnson, C., Nävert, U., Pitkäranta, J.: Finite element methods for linear hyperbolic problems, Comp. Meth. Appl. Mech. Engin. 45, 285-312 (1984).

11. Laurence, P., Bruno, 0.: Existence of 3D toroidal MHD equilibria with nonconstant pressure, Communications on Pure and Applied Math, 49, 717-764 (1996)

12. Lesaint, P.: Sur la résolution des systèmes hyperboliques du premier ordre par des méthodes d'éléments finis, Thèse de Doctorat, UPMC, Paris (1975).

13. Nédélec, J.C.: Mixed finite elements in $R^{3}$. Numer. Math. 35, 315-341 (1980). 